\title{
The Decomposition-combination Structure of the
}

\section{Chu Art in the Visual Field of Liuguan}

\author{
Yitao Zhou \\ The College of Art, South-Central University for Nationalities, Wuhan 430074, China \\ E-mail: 1xy87306548@sina.com
}

\begin{abstract}
The writing technique of the decomposition-recombination structure in the arts of the Chu state is based on the aesthetic orientation of Tao and the aesthetic attitude of "Liuguan", which has constructed the aesthetic approach different from the other types of art. The aesthetic control of "Liuguan" has produced the artistic favor and flexibility. This text focuses on the formation bases of the decomposition-recombination structure and gives a further discussion of its unique features of the arts of the Chu State.
\end{abstract}

Keywords: Tao, Liuguan, The art of Chu state, Decomposition-combination structure

\section{The aesthetic orientation of Tao}

The Chu state in the late Spring and Autumn period had conceived the Taoist school, which has produced a vital impact on the Chinese culture. Lao Tzu, the representative, and his Moral Classics have affected the spirits of many generations. Setting aside the barrier of the time, Lao Tzu of the East and Hegel of the West have something similar on the issue of the world primitive. The former thought that Tao is the primitive of the universe while the latter used absolute idea to refer to the world existence. In view of this, Hegel, the great master of Western philosophy, to some degrees, is the successor to Taoism, which lasts thousands of years. Hegel once said that the thought of Lao Tzu is extremely rich and Lao Tzu is the representative of the spirit of the ancient East. Nietzsche also said that the thought of Lao Tzu "is like an unexhausted well full of treasures. It is easily to get with a barrel". (Xiong). Tao is the core of Lao Tzu philosophy. In his eyes, Tao has no distinct forms. It is a transcendent ontology, which creates the universe. That is to say, it is the intangible Tao that gives life to the numerous things.

Tao has produced a far-reaching influence on the way by which the ancient $\mathrm{Chu}$ people viewed and grasped beauty. The bizarre imagination and the romantic artistic demonstration uplifting life freedom are all inspired from Tao. Tao belongs to the scope of sense. However, it is far beyond what is sensed. When the Chu people witnessed the aesthetic object, they let their instant intuitive feelings tune in to the profound inner world, tasting to get the various provisions from the invisible Tao out of the external world. So people can appreciate the spontaneous freedom and comfort in the imagination of beauty. The Chu people used their free soul to feel various images in the experiential world and expanded their inner association to boundless imagination to conceive an artistic pattern with the co-existence of nature, the earth and man to indicate the realm where the werden of all the things and the surging emotion have clashed then integrated. In such artistic atmosphere, it is not difficult to understand the beauties in the Odes of Chu, holy but odd, who were all in love with the ordinary people. It is also easy to understand the jamais vu for some strangeness of the dermatoglyphic ornamentation of the Chu's lacquer ware after it is decomposed and recombined. It is much easier to grasp that the unusual artistic expression is based on the affirmation of the spirit freedom.

As to how to materialize the objects of aesthetic concern into the artistic works, Lao Tzu's idea, "Doing Nothing Will Lead to Accomplishing Anything", is of the guiding significance of art practice. Mr. Chen Guying once interpreted it as "if there is no chutzpa, nothing cannot be achieved". (Chen, 1984, p.34). Indeed, to achieve some purposes, people are on one hand subject to the law. But on the other hand, they should do something active to break the bondage to display the existence of the creation. The lifelike Chu art is from the recognition by observing the universe. However, the Chu people believed that the morphology of beauty is not only the outer aims but the inner pursuit for freedom that people have sought. They are inseparable and exist in each other. The natural aesthetic approaches make the Chu people's aesthetic concern not subject to the world images. Although the fantastic concern does not expect to reproduce the real natural world, any irrelevant artistic expression will not be accepted by the Chu people. The "Doing Nothing" with a purpose and "Undoing Nothing" with deliberation make the Chu art put the inherent emotional conflicts on the new re-constructed images. The new morphology is not the representation of the natural form but the isomorphism of the heart or imagination and the natural images. Therefore, the Chu people had pursued the freedom of inner experience in the inevitability of the provisions and the self-realization without violating the laws of the nature. Different from the 
concept of freedom of the classical Greek art in the same historical period, the way by which the Chu art reproduced the external world is not to confirm man's degrees of freedom by vivid material form but to concern the world with their cheerful emotion. Tao had affected the aesthetic concern of the Chu people while the aesthetic approach of Liuguan had further deepened its existence.

\section{The basis of "Liuguan"}

We can know the meaning of "“Liuguan"” by interpreting its literal meaning. "Guan" has its other implication if going back to its origin. In the ancient times, it refers to the high-terrace buildings. It has evolved and lost its meanings as an architectural term. It is one of the ways to stand at a Guan, the viewing building, and overlook the things of the world. But here "Liuguan" has nothing direct with that. But ascending a height to enjoy a distant view will undoubtedly have some influences on the formation of "Liuguan". Its object lies "in the boundless universe and among the rich categories of things". (Wang, 1994, p.248). So the approach of viewing in the visual field of "Liuguan" has its basic features of dynamically grasping the aesthetic object in the whole world of images.

Let's go back to talking about the writing noumenon of the Chu art. The "Liuguan" of free spirit gives direct impact on their aesthetic object. The Chu people in a visual field of the heaven and the earth sought the performance of the beauty in the rheological universe. They did not focus on individual things but grasp the universe in the changes. There are words in Li Sao, saying that "I have visited everywhere and traveled all the heaven then come down to the man's world". The Nine Chapters writes that "I have overlooked as far as I can and tried to view everywhere." These lines express that the Chu people had shaken off the fetters of the specific matters with their gnosis for the life transference and free inner experience. Apparently, while viewing the world images, the aesthetic perceptual form is larger than the congregation of the images of the sense. The so-called "Liuguan" refers to the aesthetic control not confined to individual things. It has brought a free space for the Chu art creation. Thus, the internal imagination of Chu people had gained catharsis to the hearts' desire.

Wang Yi of the East Han dynasty in his Preface to Jiu Ge wrote that "Chu is a country where people believed in gods and temples. When festivals were held, they would sing and dance to please gods". (Hong, 1994, p.248). The original witchcraft filled the entire country. When on the festival of offering sacrifice, the Chu People got the emotional catharsis in the ritual dance thought they were still rational. It is far different from the worship and cult of the complete barbarism in the ancient times. "They are the Chu people, who believe in gods and themselves too. They thought that they could communicate with the spirits or gods. So despite living in a world full of spirits or ghosts, they usually felt comfortable and at ease." (Zhang, 1987, p.119). In such a mythical atmosphere with strong passion and reason, various restrictions had disappeared and the Chu art had got its free state of artistic creation.

Living with a strong idea of witchcraft and myths, the Chu people, in order to create a free inner world, made all their material wishes and expectations which reflected the internal emotional experience away from the specific things in the experiential world, regardless of the specific images they tried to copy, to express their free wills by means of the endlessly rheological nature. It is different from what Confucius disciplined that individual purpose should be realized according to $\mathrm{Li}$, the ritual. The logical starting point for the Chu people to understand the world is based on "the coexistence of nature, the earth and man and the unification of all the things and man". (Chuang, 1982, p.30). The Chu People do not attempt to separate man from the things in the rheological universe. The purpose of man's exploring the natural origin is that man and nature can coexist more harmoniously. Chuang-Tzu said that "to travel beyond the world by taking clouds and riding the sun and the moon." (Chuang, 1982, p.30). Chuang-Tzu fully affirmed that man had the same unlimited power as the universe. Moreover, he was more affirmative about the ambition to travel the world in an unconstraint way. This is the aesthetic attitude of Liuguan and the basic direction of the Chu artistic creation. It goes without saying that although the Chu people have the same power as the universe has, they do not mean to fight against nature but to harmonize the unlimited vitality with all things in the universe to confirm the omnipotent artistic creation. It is easy to see that it is only in the field of art creation that the Chu people had achieved their purpose by means of the mighty of nature which they couldn't get on their own. The Chu art is full of the internal free spirit and external natural forces, just like Yin and Yang supplementing each other, as confirms the active and enterprising spirit of the Chu art. Therefore, when the Chu people sought for the aesthetic object, they had never pay the aesthetic attention to some static and specific objects. All that could reflect the details of life, the endless weather changes could be used to build the palace hall of the Chu art.

To sum up, when the Chu people appreciated and grasped the aesthetic object, they wouldn't be fettered by the patriarchal ritual. If they were interested in the humankind, they would never take into account the grading system and put life in the ritual order to make it function like a clock. The Chu people felt the freedom of life when constructing the integration of man and nature. Their original, spontaneous, and free spirit made people adopt the aesthetic attitude of Liuguan while they perceived the surrounding, employ the way of apprehending and imagining to get the subject matters in the real life, and use little pure and rational in-position viewing to grasp the object. Therefore, aesthetic Liuguan has laid the formation bases for the Chu art. 


\section{The visual show of Liuguan}

The aesthetic sense of Liuguan has permeated with Chu art. The decomposition-combination structure is the unique writing technique of the Chu art, especially its ornamentation of lacquer ware. It did not follow the approach that Shang and Zhou dynasties adopted to deal with the lacquer ware and bronze ornamentations. They decomposed and recombined these ornamentations, then arranged the individuals according to the space and shape of the utensils. Take the ornamentation of phoenix in the Chu lacquer ware as an example. The old ornamentations of the phoenix are discomposed, the head, the two wings left linking to the traditional decoration. The re-combined emblazonments do not completely discard the old pictures, which is the basis of the decomposition-recombination structure. The new emblazonments display the vehemence of flying in the air by means of geometric vortex. Meanwhile, the decomposed phoenix is partly integrated into the abstract ornamentations to express new meanings. The special distribution does not follow the common symmetry in bronze, or adopt the casual uncontrolled padding, but form the new and flexible pictures according to the size of the space. It is like a kaleidoscope. The real prototype is broken, fragmental but colorful. As long as another angle was turned to, the broken pieces will again become another new shape. Obviously, it has de-familiarized the "alienation" that people have known well, as makes people feel exotic but fresh out of the numerous constraints, and enter a vast and broad realm full of emotional freedom, indicating that people can display their capacities of easily dealing with the world. Therefore, in the recombined forms, the abstract vehemence can be seen in the emblazonment discomposed-recombined. And the ever familiarized concrete images can be viewed in the abstract whole. In the recombined forms, the abstract whole and the partial concrete images are integrated to produce a cosmic sense of that the universe is filled with atmospheric cyclotron and life freedom. From the overall situation, the decomposition-recombination structure has the daring and ambition of holding everything and seeking the integration of the whole and the part in the rheological change. As far as the details are concerned, it thoroughly gets rid of the fetters of imitation, blurs the boundaries of reconstruction and manifestation, and highlights the expressive capacity of emotion. With the aesthetic control of Liuguan, the boundaries of nature, earth and human are well eliminated. The aesthetic pleasure it has produced is not phobic or painful, thought accompanied by wonderment. It has least the religious and mysterious meaning in the "nobleness" admired by the West. It is a kind of beauty, encouraging and high-spirited, which clearly affirms man's freedom and greatness.

What is interesting is that the tiger-seated flying bird as a woodcarving similarly demonstrates what the decomposition-recombination structure functions in the three-dimensional modeling. According to Feilian, God of wind, the integration of the three has symbolic meaning established by usage. "Why the bird and the beast is used to indicate the wind is that the wind has the attribute of a bird and a beast. Birds can fly and so does the wind. Why the wind is said to have some bestiality or be of the same type of the tiger is that it is sometimes fiery and can destroy the houses. The antlers are stuck on the flying bird which symbolizes God of Wind, signifying the lucky wind.”(Guo, 1991, p.187). As how to turn these implied meanings into the artistic entity, the decomposition-recombination structure becomes the most appropriate way. Only through examining the modeling images, the tiger and bird are integrated organically by the thinking of decomposition and recombination. The bird is standing on the back of the tiger with cloud pattern. Such a martial and soared big bird is of symbolic meaning and embodies rich imagination. However, what is incredible is that the strange and mysterious bird wings are made with antlers full of tensile force. The two antlers are stuck in the bird, seeming to be flying in the sky. So they have more visual appeal than the ordinary bird wings. Although the tiger-seated flying bird has the definite moral established by usage, how to model is a question the composer must confront with. The bird, the tiger and the antlers, irrelevant one another, are put together. Any carelessness must result in farrago. It needs the coordination of the law of the decomposition-recombination structure to reconstruct them with great imagination. Replacing the wings of the flying bird with the antlers is not the rigid regulation but the need of the decomposition-recombination structure because the real antlers keep the permanent existence of the original form. When the antlers are struck in the bird, they have displayed a more magnificent new image, soaring into the sky. From the angle of pure modeling, if the tiger-seated flying bird is fitted with the bird wings, except that big space is occupied, there is no room for imagination left, thus losing the mysterious connotation of spiritualism. The antlers have got rid of the perplexity of the physique space. The original bird wings have been reduced to the minor importance and they only played a role of balance. Therefore, the antlers are the substitute of imagination, and of the unexpected wonderful visual effect.

Although there are the same ornamentation of the phoenix and the same technique of decomposition-recombination structure, the woven and embroidered silk in No.1 tomb of Chu in the Warring States, found in the Mashan of Jiangling, has other different interest and charm. Due to the different materials and creating techniques, although the same creative approach is adopted, different types of style will be produced. From the overall layout, the woven and embroidered ornamentation of the silk mostly adopted the symmetrical composition, reflecting an identical typal tendency. But there is some individual modeling of the ornamentation demonstrating the features of the decomposition-recombination structure. In the seventeen decorative patterns of phoenix, except the head, there are almost no similarities in the other parts, as has attracted the eyeballs of many people. There are phoenixes with three heads and spread wings, or with 
abstract geometric ornamentations of double wings and double legs. And there are bird patterns out of the other decorative patterns. Such a type of things can be summed up by the way of recombination. Actually, it is only by the way that imagination can be shaped into the reality. Thus, the Chu people had maintained the aesthetic attitude of Liuguan while they used the technique of the decomposition-recombination structure.

To sum up, the decomposition-recombination structure does not includes all the artistic methods of creation. However, it fully displays the characteristics of the aesthetic Liuguan of the Chu people. The phoenix ornamentations of the three different types prove that it is not hard to find the decomposition-recombination structure which can change imagination into the graphic symbols does not purely depend on Liuguan. When "to see" is changed into "to be seen", to control the aesthetic object by means of Liuguan is the basis for the decomposition-recombination structure. Therefore, as a unique artistic technique, the decomposition-recombination structure is inseparable from the viewing paths.

\section{References}

Chen, Guying. (1984). Notes and Review to Lao Tzu. Chinese publishing house, p.34

Guo, Dewei. (1991). Explaining the tiger-seated flying bird. In Peng De (Eds.), Studying the Chu arts. Hubei Fine Arts Publishing House, p.187

Wang, Xizhi. (1994).Preface to the orchid pavilion collection. In Wu Chucai and Wu diaohou (Eds.), The Essence of Classical Chinese. Hainan publishing house, p.248

Wang, Yi. (1983). Sentences in Jiuge II. In Hong Xingzu, Complementary notes to Chu poetry. Chinese publishing house, p.55

Xiong, Chunjin. Chinese Forum, Notes to three-character primer on moral and Tao II. Noted by hermit Baiben.

Zhang, Zhengming. (1987). Cultural History of Chu. Shanghai people's publishing house, p.119

Chuang, Zhou. (1982). Equality of Things II. In Cao Chuji, Notes to Chuang Tzu. Chinese publishing house, p.30 